\title{
Ueber die Accommodationsbewegung im menschlichen Ohr.
}

\author{
Von
}

V. Hensen, Kiel.

Es kann, wie ich glaube, nicht fehlen, dass von älteren Forschern schon ausgesprochen sein muss, die Muskeln des Cavum tympani hätten dem Lauschen zu dienen. Ich habe mich vergeblich bemüht, solche Aussagen zu finden. Mach trat 1863 (1) in seiner Arbeit: "Zur Theorie des Gehörorgans" auf Grund mathematischer Betrachtungen für diese Art der Function der inneren Ohrmuskeln ein. Er sagt u. A.: „Ich glaube, dass diese (die Ohrmuskeln) so wesentlich sind wie für das Auge der Accomodationsmechanismus, dass dieselben beim aufmerksamen Hören fortwährend in Thätigkeit bleiben, dass man mittelst ihrer variirenden Spannung Töne so fixirt und verfolgt wie mit dem Auge Raumpunkte und Bewegungen. 1865 (2) gibt er ein Experiment, auf das ich zurückkomme, zur Erhärtung seiner Ansicht an, aber 1872 (3) lässt er auf Grund negativ ausgefallener Versuche seine Ansicht wieder fallen. 1878 (4) glückte mir für den Hund und die Katze der Nachweis, dass sowohl Tensor tympani wie M. stapedius sich bei Eintritt eines Schalles kurze Zeit hindurch zusammenziehen, der Tensor bei höherem Schall stärker als bei tiefem Schall. Bockendahl zeigte 1880 (5), dass die Bewegungen äusserst klein sind, entsprechend der eigenthümlichen Anordnung des Muskelgewebes. Pollak bewies 1886 (6), dass der Reflex durch den N. acusticus verläuft, und bestätigte die bezüglichen Thatsachen. V. Hammerschlag wies 1899 (7) in einer vorzüglichen Arbeit die Bahnen in der Medulla oblongata nach, die bei dem Tensorreflex eingeschlagen werden. Er (8) wirlerlegte ausserdeın in so gründlicher Weise einige meinen Versuchen entgegenstehende Beobachtungen und Ansichten von Ostmann, dass es sich erübrigt, diese zu berücksichtigen.

Das Trommelfell und wohl auch das Ringband des Stejgbügels sind nicht gespannt, sondern werden durch ihre Steifigkeit in Lage E. Pflüger', Archiv für Physiolcgie. Bd. 87. 
gehalten. Bei dem geringsten Druck von innen her auf das Manubrium mallei wirft das Trommelfell feine, im Sonnenlicht leicht zu erkennende Falten. Beim Lauschen weiss man in der Regel nicht, auf welche Tonhöhe zu rechnen ist; daher müssen alle Spannungen der Reihe nach durchlaufen werden, damit die Membranen, wenn die richtige Spannung durchlaufen wird, die Schwingung aufnehmen können, wo sie sie dann eine Weile beibehalten, auch wenn die Spannung sich ändert. Dazu kommt vielleicht, dass die Gelenke der Gehörknöchelchen-Kette etwas dichter schliessen, wenn die Muskeln ihre Arbeit leisten.

Für diese Auffassung der Thätigkeit der Muskeln beim Menschen habe ich folgende Beweise.

Wenn man eine Stimmgabel auf Resonanzkasten tönen lässt, (ich habe meistens Gabeln zwischen 400 und 1000 v. d. benutzt, übrigens auch sonst mancherlei Töne und Instrumente), wenn man dann zugleich ein Metronom schlagen lässt - 40 bis 60 Schläge die Minute - so hört man mit grosser Deutlichkeit einige Zeit nach dem Metronomschlag den Ton sich verstärken, dann bald wieder abschwellen. Es scheint mir, dass der Ton etwas rascher wächst, als wie er abfällt. Hält man das Metronom an, so tönt die Gabel mit grosser Gleichmässigkeit weiter. Steigert man die Geschwindigkeit des Metronoms auf 200-208 Schläge die Minute, so verwischen sich die Verstärkungen sehr, doch bleibt noch ein kleiner Rest bemerkbar. Der Vorgang läuft also recht langsam $a b$, er erfordert mindestens $0,3^{\prime \prime}$, um sich voll zu entwickeln. Gibt man Schläge auf Holz oder Metall in unregelmässigen Zwischenräumen, so folgt auf jeden Schlag die Tonverstärkung, wie es scheint, noch langsamer als auf Metronom oder Pendelschlag, vielleicht weil das Geräusch unerwarteter kommt. Das Geschehen ist verwickelt. Nach den Erfabrungen am Hund lässt sich mit grösster Währscheinlichleit erwarten, dass der Schlag eine reflectorische Contraction hervorruft; dann wird wohl der durch den Schlag übertäubte Ton, sobald der Schlag verklungen ist, seinerseits eine Contraction hervorrufen; daraus lässt sich der erhebliche Zeitverlust erklären. Die Aufmerksamkeit ist wesentlich auf den Ton gerichtet, aber dies geschieht nicht so plötzlich, dass es die Verstärkung erklären könnte. Eine Contrastwirkung könnte mitspielen, es muss daher versucht werden, diese Möglichkeit zu eliminiren.

Wenn man zwei verschieden hohe Stimmgabeln, von etwa 1000 
und 400 v. d., gleichzeitig erklingen lässt, so verstärkt sich vorübergehend jede, wenn man die andere plötzlich zum Schweigen bringt. Die Verstärkung ist etwaş verspätet; Contrastwirkung ist ausgeschlossen. E. Ma ch hat am Fortepiano schon ähnliche Beobachtungen gemacht und hat sie als Accommodation gedeutet; die schwebende Verstärkung tritt bei seinen Versuchen jedoch nicht so deutlich hervor wie bei den Stimmgabeln, wenn man einen c-Dur-Accord anschlägt und dann die Taște des Grundtons loslässț, ertönt das e, aber ich höre weniger die kurz dauernde Anschwellung des Tons, wie ich sie als Folge der Accommodationsbewegung erkenne, als ein continuirliches Hervortreten des e resp. des g.

Der Versuch lässt sich noch weiter vereinfachen, indem man die Mitbewegung benutzt. Wenn man dureh Innervation des $\mathbb{N}$. facialis die Nasenflügel schliesst, so hört man gleichzeitig die Verstärkung des Stimmgabeltons, vorausgesetzt, dass der Ton nicht schon leise geworden ist. Dieselbe Erscheinung gibt die Innervation der Kaumuskeln. Es scheint mir, dass beide Bewegungen zugleich ausgeführt die Tonverstärkung etwas energischer machen, aber ich bin dessen nicht sicher.

Ich habe diese Erscheinungen auch der internationalen Physiologenversammlung in Turin zu Gehör gebracht. Gelegentlich der Discussion erklärte Gad, dass er seine Binnenmuskeln des Ohres willkürlich zu innerviren vermöge; er habe den Versach gemacht, während die Stimmgabel (1000 v..d) geklungen hábe; und theile mit, dass er bei starker Innervation zwar nur ein Mủskelgeräusch höre, aber bei schwacher Innervation dieselbe Verstärtiung. des. Tons erhalte wie bei meinen Versuchen.

Nach Allem, was wir nunmebr wissen, erscheint gesichert, dass die beobachteten Empfindungen auf Accommodationsbewegungen im Ohr beruhen. Die Hinwendung der Aufmerksamkeit kann dabei nur wenig in Betracht kommen; bei dem Innervationsversuch ist sie getheilt, und bei dem Metronomversuch kann man seine Gedanken etwas abschweifen lassen, ohne die Verstärkungen zu verlieren.

Es gibt noch eine Erfahrung, die vielleicht hierher gehört. Urbantschitsch (9) und später Hessler (10) haben gefunden, dass bei dem Abklingen der Stimmgabel der Ton verschwinden und dann noch ein oder zwei Mal wieder auftreten kann. Der in psychologisch-akustischen Erscheinungen gethbte Assistent des Instituts, Dr. F. Kr ü ger, und ich haben diese Erfahrung nicht nur als objec- 
tive Erscheinung bestätigt, sondern namentlich auch festgestellt (mittelst einer Art Wellensirene), dass bei schwächer werdendem, continuirlich sinkendem Ton die gleiche Erscheinung auftritt und bei Bestimmung des akustischen Schwellenwerths sehr störend wird. Diese Schwankungen des Schwellenwerthes wären vielleicht auf die Accommodation zu beziehen. In diesem Falle müssten aber die Muskeln etwas längere Zeit in richtiger Spannung stehen bleiben. Das thun sie für gewöhnlich nicht, aber bei dem Lauschen auf sehr Leises wäre diese Art der Thätigkeit denkbar. Man kennt auch von anderen Sinnesorganen Schwankungen der Empfindung in der Nähe der Schwelle, die Deutung bleibt daher zweifelbaft.

Die Mitbewegungen der inneren Ohrmuskeln sind u. A. schon von L. Fick und Lucae beobachtet; ich habe in meiner Physiologie des Gehörs (11) darüber und über die entsprechenden Untersuchungen am Trommelfell berichtet. Selbst sehr genaue Beobachtungen, die Mach und Kessel (3) am schwingenden Trommelfell, während die Versuchsperson auf den bezüglichen Ton lauschte, angestellt haben, führten zu keinem entsprechenden Ergebniss, doch haben sie festgestellt, dass beim Lauschen auf lauten Ton das Trommelfell nicht gespannt bleibt. Die Misserfolge beruhen wesentlich darauf, dass. eine dauernde und nicht eine rasch vorübergehende Wirkung erwartet wurde; ausserdem sind die Bewegungen bei der Accommodation sehr klein, und wenn die Töne so sehwach werden, dass man darauf lauschen muss, wird es nicht mehr möglich sein, Schwingungen zu sehen, folglich auch nicht, wenn in diesem Falle dauernde Contractionen eintreten sollten.

Die Hypothese von J. Müller, dass die Muskeln ein Schutzapparat des Gehörs seien, ist unhaltbar. Sie beruht auf richtigen Versuchen von Savart (12), nach denen eine Membran durch Spannung taub gegen tiefe Töne wird. Gefahrdrohend für das Gehör sind notorisch in erster Linie starker Knall und die Schläge beim Kesselschmieden. Würde ein Schutzengel, ehe der Knall das Ohr trifft, eine Contraction des oder der Muskeln veranlassen, so würde damit falsch operirt werden, denn eine gespannte Membran wird durch einen Stoss leichter zerrissen als eine nicht gespannte, weil die Spannung sich zu der Kraft des Stosses addirt.

Nachdem ich in Turin obige Beobachtungen hatte anstellen lassen, wurde mir nachträglich mitgetheilt, dass auch dann die Tonschwellung einträte, wenn man mit der Hand die Nase schliesse. Ich konnte: 
diese Entdeckung sogleich bestätigen. Als dann ein anderer Herr den Facialisversuch anstellte und ich ihn dabei beobachtete, ergab sich, dass ich fast in demselben Augenblick die Tonschwellung hörte, wie er sie haben musste. Weitere Prüfungen ergaben, dass auch andere Personen die gleiche Erfahrung gewannen. Die Versuchsperson muss indessen ersucht werden, gleichzeitig auf die Gesichtsbewegung und den Ton zu achten; in dem Falle kann sie die Schwellung selbstständig entdecken, sonst kann die Empfindung unbemerkt bleiben.

Es ergibt sich weiter, dass schon eine energische Contraction der Hand die Reaction hervorruft; selbst bei einer Zusammenziehung des Fusses erhält Dr. Krüger die Schwellung, während ich sie dabei nicht mehr deutlich erhalte. Demnach ist für die Wirkung des Zudrückens der Nase mit der Hand eine zweifache Erklärung.möglich: sie kann Folge eines Reflexes von der Nasenhaut, aber auch Folge der Handbewegung sein. Es ist ferner, - worauf mich $\mathrm{Kr} u ̈ \mathrm{~g}$ e r aufmerksam machte, - möglich, dass eine unbewusste Mitbewegung eintritt, während man die Bewegung des Anderen beobachtet. Zu erwähnen ist noch, dass eine Patientin mit beiderseits fast zerstörtem Trommelfell bei dem Metronomversuch, den ich den Otiatern glaube empfehlen zu dürfen, eine Veränderung des Tons angab, ohne aber angeben zu können, worin diese Veränderung bestand.

Ob die Muskeln, z. B. in einem Concert, fortwährend in Bewegung sind, oder ob sie sich an den Tonwechsel gewöhnen resp. sich dagegen abstumpfen, dies zu entscheiden finde ich kein Mittel. Die Langsamkeit der Reaction whirde bei rascher Tonfolge eine andauernde, aber in jhrer Stärke wechselnde Zusammenziehung der Muskeln bedingen. Da die Augenmuskeln, namentlich der Levator palpebrae, den ganzen Tag zu arbeiten pflegen, ist von etwa übergrosser Ermüdung nichts zu fürchten.

Diese Dinge dürften noch viele Studien erfordern; leider wird in nächster Zeit in dem hiesigen Institut die Angelegenheit nicht weiter verfolgt werden können. 
360 V. Hensen: Ueber die Accommodationsbewegung im menschl. Ohr.

\section{Lit er at ur.}

1) E. Mach, Zur Theorie des Gehörorgans. Sitzungsber. der Akademie Wien. 16. Juli 1863.

2) Derselbe, Bemerkungen über die Accommodation des Ohrs. Ebenda S. 343. April 1865 .

3) E. Mach und J. Kessel, Versuche über die Accommodation des Ohrs. Ebenda. October 1872.

4) Hensen, Beobachtungen über die Thätigkeit des Trommelfellspanners. Arch. f. (Anatomie u.) Physiologie 1878 S. 312.

5) A. Bockendahl, Ueber die Bewegungen des M. tensor tympani. Arch. f. Obrenheilkunde Bd. 16 S. 1.

6) Pollak, Ueber die Function des M. tensor tympani. Medic. Jahrbücher S. 555. Wien 1886.

7) $\nabla$. Hammerschlag, Ueber die Reflexbewegung des M. tensor tympani und ihre centralen Bahnen. Sitzungsber. d. Akademie Wien. 12. Jan. 1899, und Arch. f. Ohrenheilkunde Bd. 47 S. 251.

8) Derselbe, Arch. f. Ohrenheilkunde Bd. 46. S. 1 u. 89.

9) V. Urbantschitsch, Ueber eine Eigenthümlichkeit der Schallempfindung geringster Intensität. Centralblatt f. med. Wissenschaft $1875 \mathrm{Nr} .35$.

10) Hessler, Beitrag zur Physiologie. Arch. f. Obrenheilkunde Bd. 18 S. $234 \mathrm{ff.}$

11) Hensen, Physiologie des Gehörs, in Hermann's Handbuch der Physiologie Bd. 3 Th. 2 S. 62.

12) Savart, Journal de Physiologie Bd. 1 S. 183. 1824. 